\title{
Linx
}

Revue des linguistes de l'université Paris X Nanterre

$80 \mid 2020$

L'héritage de Jean Dubois et Françoise Dubois-Charlier

\section{Place de l'œuvre de Jean Dubois et de Françoise Dubois-Charlier dans l'histoire de la linguistique française}

The work of Jean Dubois and Françoise Dubois-Charlier in the history of French linguistics

Denis Le Pesant

(2) OpenEdition

Journals

Édition électronique

URL : http://journals.openedition.org/linx/6751

DOI : $10.4000 / \operatorname{linx} .6751$

ISSN : 2118-9692

Éditeur

Presses universitaires de Paris Nanterre

Référence électronique

Denis Le Pesant, «Place de l'œuvre de Jean Dubois et de Françoise Dubois-Charlier dans l'histoire de la linguistique française », Linx [En ligne], 80 | 2020, mis en ligne le 31 juillet 2020, consulté le 05 août 2020. URL : http://journals.openedition.org/linx/6751

Ce document a été généré automatiquement le 5 août 2020

Département de Sciences du langage, Université Paris Ouest 


\section{Place de l'œuvre de Jean Dubois et de Françoise Dubois-Charlier dans l'histoire de la linguistique française}

The work of Jean Dubois and Françoise Dubois-Charlier in the history of French linguistics

Denis Le Pesant

\section{Introduction}

1 L'immensité de la culture de Jean Dubois (1920-2015) devait moins à sa formation à la Sorbonne, philologique et classique, qu'à sa curiosité de toutes choses, surtout dans le domaine des sciences de la nature et celui de l'histoire, ainsi qu'à son intérêt pour l'actualité politique et sociale. La plupart de ses maîtres l'avaient déçu ${ }^{1}$ et il avait sans enthousiasme préparé et obtenu l'agrégation des lettres. Devenu professeur de lycée pour près de 20 ans, il prépare une thèse de doctorat sur le vocabulaire politique et social au début des années 1870, qu'il soutient en 1958. A cette époque, il a connu l'expérience du syndicalisme où se révèlent son sens de l'organisation et son art de diriger une équipe. Son extraordinaire fécondité comme lexicologue, lexicographe, morphologue, psycholinguiste, syntacticien et créateur de revues, il la doit non seulement à sa puissance de travail, à son esprit d'entreprise et à son goût de l'action, mais aussi à son attirance pour les idées nouvelles, sur laquelle je vais insister dans cet article.

2 Il devient universitaire ${ }^{2}$ aux alentours de sa quarantième année, et c'est aussi à ce moment-là que débute sa période créatrice de linguiste. Il n'y a pas de doute que les circonstances de sa vie professionnelle et privée ont joué dans ce «timing ». En effet il eut d'abord la chance, comme beaucoup d'autres, de résider à Paris à un moment, les années 1960-1980, où non seulement la linguistique est instaurée comme nouvelle discipline universitaire par le gouvernement français (cf. la réforme Fouchet de 1967) avec la création de postes que cela implique surtout après mai 1968, mais aussi où cette 
discipline, grâce à l'influence conjointe de Claude Lévi-Strauss et de Roman Jakobson et ce dans la lignée des travaux de Ferdinand de Saussure, est instaurée comme « discipline phare » des sciences humaines. Du coup Jean Dubois fit un temps partie des acteurs français du mouvement d'idées mondialement connu sous le label de structuralisme. J'aborderai cet aspect dans la première partie de cet article.

3 Il eut aussi la chance d'être le frère de Claude Dubois, directeur en chef des dictionnaires et encyclopédies Larousse pendant 30 ans, qui le fit travailler très tôt dans la maison d'édition. «Depuis 52-53, déclare Dubois à Chevalier et Encrevé (2006: 153), je travaillais chez Larousse sur les dictionnaires. Et depuis 58, on faisait le Grand Larousse Encyclopédique, en dix volumes ». Plus tard, c'est dans le cadre de ses activités chez Larousse qu'il est amené à créer Langages et Langue française, qui restent aujourd'hui des revues françaises de premier plan.

4 Il eut enfin la chance de rencontrer l'angliciste Françoise Charlier et de l'épouser. Françoise Dubois-Charlier (1941-2016) fut aussi son étudiante: il dirige sa thèse soutenue en 1970, sur l'alexie pure (cf. Dubois-Charlier 1970) et ils sont ensuite coauteurs de nombreux articles et ouvrages. Indépendamment de Jean Dubois, Françoise Dubois-Charlier a aussi à son actif de nombreux travaux. C'est grâce à leur association que de nombreux articles issus des nouvelles écoles de linguistique américaines furent diffusés en langue française, grâce à des traductions et des commentaires. Mis à la retraite, Jean Dubois rejoignit Françoise Dubois-Charlier à Aix-en-Provence, où elle avait obtenu un poste de Professeur de linguistique anglaise. Ils résidèrent à Aix jusqu'à leur mort, sans arrêter de travailler ensemble à la révision de leurs dictionnaires informatisés (cf. dans ce numéro, l'article de Guy Lapalme et Denis Le Pesant).

5 Je m'étends moins dans cet article sur les activités d'enseignant et de directeur de thèse de Jean Dubois que sur celles d'éditeur de revues. J'approfondis l'ouverture de Dubois et Dubois-Charlier aux courants nouveaux (la linguistique américaine, l'école française d'analyse du discours et la lexicographie informatisée) et leur intérêt pour les linguistiques appliquées: la didactique, la neurolinguistique et le TAL (Traitement Automatique du Langage). J'insiste surtout sur leurs activités de grammairiens, en morphologie, syntaxe et lexicologie.

6 La polyvalence de leurs activités est, on le voit, exceptionnelle. Elle est d'autant plus remarquable que ces activités sont présentées comme étroitement intriquées les unes dans les autres. Voici quelques exemples de ce phénomène. Pour faire connaître en France la linguistique américaine (entre autres de Harris et de Chomsky) avec l'aide de l'angliciste Françoise Dubois-Charlier, Jean Dubois crée et utilise la revue Langages et initie des traductions en français. Autre exemple : en publiant le Dictionnaire du français contemporain (Dubois et alii 1967), il livre au public un dictionnaire d'une conception tout à fait novatrice; il agit alors à la fois en tant que syntacticien et lexicologue tout en visant, dans une démarche pédagogique, un lectorat d'apprenants du français langue maternelle et d'étudiants et enseignants du supérieur (cf. infra Section 5.1). De façon analogue, quand Dubois et Dubois-Charlier publient leur Dictionnaire du FLE (Dubois et Dubois-Charlier 1978 et 1979), ils s'adressent nécessairement au premier chef aux didacticiens et aux apprenants du français, mais ils le font en produisant un objet lexicographique totalement novateur du point de vue théorique, comme on le verra infra dans la Section 5.2. D'autre part, en créant la revue Langue Française, Dubois vise à attirer l'attention d'un public d'enseignants et de didacticiens sur les publications grammaticales et dictionnairiques de l'éditeur Larousse, tout autant qu'à les sensibiliser 
aux idées nouvelles en sciences du langage. Enfin, ayant élaboré une base de données informatisées sur les verbes français (LVF, 1997a), Dubois et Dubois-Charlier prennent la peine d'en fournir parallèlement une version papier largement rédigée et plus aisément consultable (LVF 1997b), ce qui témoigne de leur souci de s'adresser à tout le monde.

\section{Le contexte des années 1960-1980 : la vogue du structuralisme}

7 Les années 1960 marquent le début d'un tournant linguistique, un linguistic turn. Cette notion réfère à toute période de l'histoire des idées consistant, au sein de plusieurs domaines relevant des humanités, à mettre la réflexion sur le langage au centre des spéculations. La vogue du structuralisme, en France, est un exemple typique de linguistic turn: la linguistique structurale héritée de Saussure et du Cercle de Prague (JakobsonTroubetzkoy) se voit érigée en discipline modèle pour la plupart des sciences humaines, notamment l'ethnologie (Cl. Levi-Strauss), la sémiotique (R. Barthes, A.-J. Greimas), la théorie de la littérature (T. Todorov, J.-P. Faye), la psychanalyse (J. Lacan) et la philosophie (L. Althusser, M. Foucault) ${ }^{3}$.

8 L'influence de Saussure en France a longtemps été plutôt marginale jusqu'en 1960. Toutefois elle n'a jamais cessé, grâce au linguiste français le plus important de l'époque, Antoine Meillet (1866-1936), qui avait succédé à Saussure à l'Ecole Pratique des Hautes Etudes. Le successeur de Meillet au Collège de France fut Emile Benveniste, qui contribua à la vogue du saussurianisme en France jusqu'en 1960. L'influence de Saussure s'exerça surtout ailleurs qu'en France, notamment au sein du Cercle de Prague, et surtout aux Etats-Unis. Saussure influence Bloomfield, qui est la source principale de Harris et Chomsky. D'autre part, Jakobson s'installe à New-York dès 1941 et est nommé à Harvard la même année.

9 Il n'est pas exagéré de considérer que le structuralisme français est né à New-York en 1942, à l'Ecole Libre des Hautes Etudes. C'était une institution résistante (gaulliste) regroupant quelques-uns des plus célèbres membres de l'intelligentsia, principalement française, ayant fui le nazisme, tels Gustave Cohen, Henri Focillon, Jacques Maritain, Jean Perrin, Alexandre Koyré, André Weill, Vladimir Jankélévitch, Henri Lévy-Bruhl et Jean Wahl. Parmi les membres de l'Ecole Libre des Hautes Etudes figuraient aussi Claude Lévi-Strauss et Roman Jakobson. Ces derniers y font connaissance, se lient au linguiste et ethnologue Franz Boas (que Harris range parmi ses maitres, cf. Harris 1990: 9) et préparent leur célèbre analyse des Chats de Baudelaire. Le système universel phonologique de Jakobson impressionne à ce point Lévi-Strauss que ce dernier passe pour être à l'origine du slogan de "linguistique, discipline phare des sciences humaines ".

10 Ce n'est pas ici le lieu de détailler la manière dont les différentes disciplines des sciences humaines ont été concernées par le mouvement structuraliste. En linguistique même, le mot structural a été beaucoup utilisé, y compris par Jean Dubois, sans qu'on puisse y déceler autre chose qu'une référence à la lignée de Saussure, Benveniste et Jakobson, en somme à la linguistique elle-même, par opposition à la philologie qui prévalait en France jusqu'aux années 1960. 
11 Dans la vogue structuraliste, Dubois et Dubois-Charlier ne jouissent évidemment pas de la même notoriété médiatique que les personnalités que j'ai énumérées au début de cette section. Mais, au-delà de l'écho que rencontrent auprès d'un vaste public d'enseignants et d'apprenants leurs nombreuses publications, dont plusieurs dictionnaires et les deux revues qu'ils animent, il se manifeste dans leurs activités et autour d'elles l'influence d'un certain nombre de facteurs caractéristiques de l'époque du structuralisme, à savoir :

- l'explosion de la population étudiante qui conduit à une création massive de postes à l'Université et à la fondation de nombreuses universités ;

- le linguistic turn structuraliste, qui conduit à la création d'une nouvelle discipline dans l'Université française, la linguistique ;

- le slogan structuraliste du prétendu rôle phare de la linguistique pour les sciences humaines et sociales ;

- l'esprit de 1968 qui balaie entre autres choses la domination de la philologie (commentaire historico-idéologique des textes antiques et classiques et étude du style) en Sorbonne, et qui permet les premiers succès des "non-agrégés » (cf. Chevalier et Encrevé 2006) que sont R. Barthes, A.-J. Greimas et B. Quemada ;

- la notoriété nouvelle et tardive en France de F. de Saussure et d'E. Benveniste ;

- l'écho des nouvelles grammaires américaines, notamment celles de Harris et de Chomsky; dans la promotion de ce dernier aspect, Dubois et Dubois-Charlier jouent un rôle prééminent.

\section{L'ouverture aux courants nouveaux}

L'ouverture de Jean Dubois et Françoise Dubois-Charlier aux courants nouveaux se manifeste non seulement dans leur effort d'introduire en France les nouvelles linguistiques américaines ( $c f$. l'article de Jacqueline Léon dans ce numéro), mais aussi dans leur promotion des travaux d'analyse du discours et dans leur adhésion au courant des Grammaires distributionnelles et transformationnelles de Zellig Harris et Maurice Gross.

\subsection{Analyse du discours}

13 Dès le début de sa carrière, Jean Dubois s'intéresse au discours, mais plus en tant que lexicologue qu'en tant que théoricien de l'analyse du discours: sa thèse principale, soutenue en 1962, a pour sujet : «Le vocabulaire politique et social en France de 1869 à 1872 à travers les cuvres des écrivains, les revues et les journaux». Par la suite, sans engager le cours de ses recherches dans le domaine de l'analyse du discours, il se fait l'avocat et le promoteur de recherches françaises tout à fait novatrices. Je reprends ici les informations et les analyses de Dominique Maingueneau, qui écrit à propos de Jean Dubois :

Certes, il est surtout connu pour ses travaux en matière de lexicologie ou de morphosyntaxe, mais on ne saurait oublier qu'il a aussi joué un rôle très important dans l'émergence de l'analyse du discours en France, et plus particulièrement de ce qu'on a appelé par la suite "l'École française d'analyse du discours" (...). Son rôle a en effet été essentiel pour crédibiliser ce nouveau courant de recherche. (Maingueneau 1996 : 27).

14 De fait, il n'y pas moins de 9 numéros de Langages consacrés à l'analyse du discours entre 1969 et 1986, avec des articles encore aujourd'hui beaucoup cités de J.-B. Marcellesi, D. Maldidier, M. Pêcheux, G. Provost-Chauveau et J.-J. Courtine. Jean Dubois 
a également marqué son intérêt pour l'Ecole française d'analyse du discours en favorisant le recrutement de Denise Maldidier à l'Université de Nanterre. On retrouve dans ce phénomène un écho de «l'ambiance » structuraliste de ces années, évoquée cidessus dans la Section $1^{4}$.

L'adhésion de Dubois et Dubois-Charlier au courant des grammaires distributionnelles et transformationnelles, qui remonte à Z. Harris, a pu jouer dans leur intérêt pour l'analyse du discours. Harris est en effet souvent présenté comme le fondateur de cette spécialité. Or Françoise Dubois-Charlier est l'auteur d'une traduction, parue dans Langages $\mathrm{n}^{\circ} 13$, numéro co-dirigé par Jean Dubois, d'un article de Harris (1952) intitulé Discourse analysis. Maingueneau nuance toutefois l'importance de cette influence en indiquant que Jean Dubois reproche à Harris, à juste titre, de ne pas prendre suffisamment en compte les phénomènes énonciatifs. D'après lui (cf. Maingueneau 1996 : 33) Jean Dubois, à cette époque, se serait rapidement éloigné des méthodologies structuralistes en matière d'analyse du discours, au profit du générativisme et des théories de l'énonciation.

\subsection{Evolution des idées de Jean Dubois}

Jusqu'aux environs de 1970, on remarque une certaine évolution des idées de Dubois et Dubois-Charlier, sensible à la lecture de la préface des trois volumes de la Grammaire structurale du français.

Grammaire structurale du français : Nom et pronom (Larousse, 1965)

Grammaire structurale du français : Le verbe (Larousse, 1967)

Grammaire structurale du français: La phrase et les transformations (Larousse, 1969)

17 Le terme de structural dans les titres de ces ouvrages ne semble pas faire spécialement référence au structuralisme français qui s'autorise volontiers de Saussure, Jakobson, Benveniste et Martinet : les trois premiers ne sont pas cités dans les préfaces des deux premiers tomes; Martinet est le seul à être évoqué fugitivement dans le premier volume. Quant à Saussure et Jakobson, ils ne sont évoqués que dans le troisième volume.

Dans la préface du tome 1 (1965), Dubois s'autorise de Z. Harris, en tant que successeur de Bloomfield et représentant de la méthodologie distributionaliste, et il évoque aussi Hjelmslev principalement sur la question de la relation syntaxe-sens. Harris, dit-il, aussi bien que Hjelmslev et Bloomfield, font l'hypothèse "si féconde en sémantique " de l'isomorphisme entre les deux formes. Jean Dubois repousse d'autre part l'idée d'une primauté de la syntaxe, que défendra plus tard Harris avec la notion de rupture d'équiprobabilité des événements linguistiques en elle-même porteuse d'information ${ }^{5}$. On ne s'étonnera pas que les travaux de Z. Harris aient attiré d'emblée l'attention de Jean Dubois, qui le lit avec passion et décide d'emblée de le faire traduire; en effet, Harris est à ce point connu aux USA comme en France qu'André Martinet, de retour à Paris après des années passées à l'Université Columbia de New-York, s'évertue lui aussi à diffuser ses travaux, selon le témoignage personnel de Pierre Encrevé en 2006 :

Martinet, la première chose qu'il m'avait fait lire comme étudiant, c'est le livre de

Z. Harris, l'aujourd'hui fameux Methods in Structural Linguistics; c'était obligatoire pour Martinet, ça faisait partie du programme pour tout étudiant. (Propos de P. Encrevé dans Chevalier et Encrevé 2006 : 371) 
La préface du tome 2 de la Grammaire structurale du français (1967) évoque brièvement la notion de transformation, et ce n'est que dans le tome 3 (1969) sur la Phrase et les transformations que se manifestent, de façon en quelque sorte œcuménique, les références non seulement à Saussure et au Cercle de Prague, mais aussi, sur plusieurs pages, à la grammaire générative. On ne trouve aucune référence à Maurice Gross, dont l'ouvrage majeur, Méthodes en syntaxe, ne paraîtra qu'en 1975, mais qui a déjà publié chez Larousse en 1978 sa Syntaxe du verbe.

Françoise Dubois-Charlier de son côté travaille assidument à faire connaître les travaux générativistes. On se reportera sur ce point aux articles de Jacqueline Léon et de Jacques François, dans ce numéro.

Dans la dernière étape de l'évolution de leurs idées, à partir des années 1970, Dubois et Dubois-Charlier se rapprochent de Maurice Gross et de son équipe, le LADL. Ainsi s'effectue un retour à Harris et une adhésion au programme initié par Maurice Gross d'expliciter l'extension lexicale des règles de grammaire, dont Harris avait tracé les formes : il s'agit du projet de lexique-grammaire. Dans les années 1980, Jean Dubois, sans faire partie administrativement de l'équipe du LADL, tient dans les locaux du laboratoire des réunions hebdomadaires sur le lexique des verbes français, auxquelles assistent les chercheurs, notamment Alain Guillet, et les doctorants de l'équipe. C'est dans ce cadre que s'élabore progressivement ce qui va devenir le dictionnaire des Verbes Français (cf. infra dans la Section 6.2), qui est lui aussi une sorte de lexiquegrammaire informatisé. Dans les années 1990 à 2010, Dubois et Dubois-Charlier s'efforceront d'élaborer une synthèse des travaux de toute leur vie, et ils donneront à cette synthèse la forme d'un dictionnaire informatisé: il s'agit du Dictionnaire Electronique des Mots (DEM), œuvre malheureusement inachevée (cf. l'article de Guy Lapalme et Denis Le Pesant, dans ce numéro).

\section{L'ouverture de Dubois et Dubois-Charlier aux linguistiques appliquées}

Les linguistiques appliquées dont il va être question dans cette section sont la neurolinguistique, la didactique du français et le TAL.

\subsection{Travaux sur des questions de neurolinguistique}

Jean Dubois n'a écrit, en collaboration avec d'autres auteurs, pas moins de 23 articles sur des questions de neurolinguistique, plus exactement de neuro-psycho-pathologie $d u$ langage. Le principal co-auteur de la majorité de ces articles est le professeur Henri Hécaen (1912-1983), neurologue de notoriété mondiale, et directeur un temps d'une unité de l'INSERM. Son ouvrage majeur, paru en 1949, a pour titre Le cortex cérébral, une étude neuro-psycho-pathologique. On trouvera dans la bibliographie de Dubois qui figure à la fin de ce numéro la liste complète de ces articles.

24 A certains de ces articles a collaboré Luce Irigaray (née en 1930). Linguiste, psychanalyste et philosophe, elle est aussi une figure féminine fondatrice de la French theory dans le domaine des études de genre. Selon Marcie (1993: 40), « le travail de L. Irigaray, avec sa thèse consacrée au Langage des déments (Irigaray, 1973), constitue désormais l'ouvrage de référence unanimement considéré comme fondateur pour son 
approche des désorganisations de la sphère sémantique dans la pathologie corticale dégénérative ». Cette thèse avait été dirigée par Jean Dubois. Il est remarquable de constater que l'autre thèse dirigée par Jean Dubois sur des questions de neurolinguistique fut celle, soutenue en 1970, de Françoise Dubois-Charlier sur une pathologie peu connue, l'alexie pure (cf. Dubois-Charlier 1970).

Revenons à Henri Hécaen, qui étudia en particulier les troubles du langage, du geste et de la perception. C'est dans ce cadre qu'il rechercha la collaboration de Jean Dubois. Voici ce qu'écrit Pierre Marcie (1996), lui-même collaborateur de Hécaen à l'INSERM, dans un article intitulé Jean Dubois et l'avènement de la neurolinguistique :

Henry Hécaen avait recherché le contact avec les spécialistes impliqués dans l'étude du langage, tant il lui semblait naturel, sinon nécessaire même, que l'aphasiologie déborde le seul champ de la neurologie pour être investie de préoccupations qui se situent parmi celles de la Linguistique. (...). Pendant une douzaine d'années, J. Dubois participa pleinement au développement des travaux du groupe de chercheurs constitué autour de H. Hécaen et du Laboratoire de Pathologie du Langage de l'École des Hautes Études en Sciences Sociales.

On voit par ces propos que la collaboration de Dubois avec Hécaen est loin de se limiter à la rédaction d'articles. Pierre Marcie (1996) précise :

Il participa à tous les moments de travail dans la vie d'un laboratoire de recherche. Il sut dépenser de longs moments de son temps pour assurer lui-même les examens du langage chez les sujets normaux ou les groupes de malades dans le cadre des travaux de validation de la batterie d'examen standardisé construite dans le laboratoire. (ibidem p. 37-38) (...). Il fut de ceux qui entraînèrent l'adhésion de $\mathrm{H}$. Hécaen pour la confection d'un examen standardisé et étalonné comme mesure indispensable pour étayer et conforter la seule et immédiate intuition clinique (p. 39).

L'élément qui cimenta la relation entre le neurologue Henri Hécaen et le linguiste Jean Dubois fut l'œuvre de Roman Jakobson (1896-1982). Ce linguiste joua un rôle fondamental dans la genèse du mouvement structuraliste en tant que fondateur de la phonologie, méthode de classification des phonèmes de toute langue en fonction d'un système de traits distinctifs s'opposant de façon binaire ( $c f$. supra Section 1). Mais Jakobson est aussi l'auteur d'une typologie des aphasies, dans une recherche qui le conduisit à postuler l'existence des deux axes fondamentaux qui structurent selon lui le langage, l'axe syntagmatique et l'axe paradigmatique, et à en déduire, dans le domaine de la poétique, l'existence des figures homologues de la métonymie et de la métaphore. Henri Hécaen faisait grand cas des travaux de Jakobson sur l'aphasie ${ }^{6}$, et c'est en tant que spécialiste de Jakobson tout autant que spécialiste du langage en général que Dubois fut sollicité par Hécaen pour participer aux travaux du Laboratoire de Pathologie du Langage de l'EHESS.

Quant à Françoise Dubois-Charlier, elle défend, comme l'écrit Jacques François (dans ce numéro) « la dimension neurolinguistique aux côtés de son mari et de Henry Hécaen (cf. le numéro 25 de Langages, 1972 intitulé Neurolinguistique et neuropsychologie) et en coordonnant (en collaboration) un numéro entier de la même revue $\left(n^{\circ} 44,1976\right)$ sur l'alexie, où elle publie elle-même un article (cf. Dubois-Charlier 1976).

\subsection{Linguistique théorique et pédagogie}

Le fait d'avoir lancé en 1968 une revue de linguistique, Langue Française, s'adressant de façon privilégiée à un lectorat d'enseignants, et le fait d'avoir publié, souvent en 
collaboration avec Françoise Dubois-Charlier, tant de travaux théoriques à visée partiellement applicative à la pédagogie, illustrent l'intention de Jean Dubois de faire en sorte que les travaux de linguistique théorique soient utiles aux enseignants et aux apprenants. Dans une déclaration datée de 1971 et citée par Franck Marchand, Jean Dubois exprime cette conviction :

La linguistique n'est pas seulement une étude théorique; elle a un champ d'application ; c'est celui des langues vivantes et celui de la langue maternelle, pour nous le français. Voilà pourquoi la linguistique (...) est aujourd'hui au centre des problèmes de l'enseignement moderne du français. (Franck Marchand, 1996 : 45)

Sur des questions de pédagogie, Jean Dubois intervient lui-même dans Langue Française en tant qu'auteur et éditeur de numéro: à l'occasion du numéro 5 (Linguistique et pédagogie, Dubois et Sumpf 1970) et du numéro 14 (Linguistique, formation des enseignants et enseignement supérieur, Dubois 1972). D'autre part, il publie en 1973 avec René Lagane trois livrets pédagogiques rassemblés sous le titre de Comment apprendre la grammaire. Françoise Dubois-Charlier participe à ces travaux de vulgarisation à visée pédagogique en produisant avec d'autres auteurs, Danielle Leeman, Simone Delesalle et Raoul Rossi, des films d'animation et des livrets pédagogiques. D'autre part, elle signe seule ou en collaboration un certain nombre d'ouvrages pédagogiques sur l'anglais.

Mais les travaux linguistiques en eux-mêmes, selon Jean Dubois, ne doivent pas être confondus avec les travaux pédagogiques. Il distingue entre le discours de la grammaire « scientifique » et un deuxième discours, celui de la grammaire pédagogique qui, écritil en 1972 dans Langue Française 14, «ne peut s'identifier ni à la grammaire scientifique, ni à l'amalgame confus qui existe actuellement». Contre cet "amalgame confus » qui caractérise certaines pratiques et publications pédagogiques de l'époque influencées maladroitement par la linguistique, les propos de Jean Dubois sont sévères ${ }^{7}$.

Il n'en reste pas moins que le souci de Dubois et Dubois-Charlier que leur travaux servent les besoins des apprenants sera constant, jusques et y compris dans les domaines de la grammaire et de la lexicologie théoriques, à savoir dans la Grammaire structurale du français (1965 et 1967), les Eléments de linguistique française (avec Françoise Dubois-Charlier, 1970), La nouvelle grammaire du français (avec R. Lagane, 1973) et, pardessus tout, dans le Dictionnaire du français contemporain (DFC, avec R. Lagane 1967).

Le Dictionnaire du Français Contemporain s'est bien vendu. Je ne sais pas évaluer son impact sur les enseignants du premier et du second degré, mais il est avéré en revanche qu'il a largement été utilisé dans les départements universitaires de lettres et de linguistique. L'ouvrage est extrêmement novateur du point de vue théorique. Toujours du point de vue lexicographique, les deux tomes Dictionnaire du français langue étrangère (1978 et 1979) par Dubois et Dubois-Charlier doivent être cités en tant qu'œuvres destinées de façon privilégiée à des enseignants. Mais il s'agit aussi, comme dans le cas du DFC, de travaux extrêmement novateurs du point de vue théorique. Je reviendrai sur ces deux ouvrages infra aux Sections 5.1 et 5.2.

\subsection{Traitement automatique du langage (TAL)}

S'étant rapprochés de Maurice Gross et du LADL dans les années 1970-1980, Dubois et Dubois-Charlier ont eu à cœur d'élaborer le dictionnaire Les Verbes Français (plus de 25000 entrées) sous la forme initiale d'un dictionnaire informatisé (cf. Dubois et 
Dubois-Charlier 1997a). Dans la préface de la version imprimée de l'ouvrage, Dubois et Dubois-Charlier (1997b) présentent la version informatisée de LVF en ces termes :

Ce dictionnaire informatisé, se comportant comme un hypertexte, permet deux types principaux d'opérations :

1. des tris croisés entre les diverses rubriques permettent de répondre à un nombre indéfini de questions; en voici quelques-unes parmi celles que je me suis posées :

Quelles sont les constructions syntaxiques dominantes dans un domaine comme la Géologie ? (...)

Quelles sont les classes de verbes à sujet humain, transitifs indirects avec préposition à ou de?

Quelles sont les classes où l'on trouve le plus de verbes à sujet humain relevant de la langue populaire de type clamser (...). Se révèlent dominantes les classes $\mathrm{P}$ (psychologie), $\mathrm{H}$ (comportement), M (mouvement)

Le complément en avec est-il caractéristique de certaines classes sémantiques? Il se trouve principalement dans les schèmes syntaxiques des classes C (communication) et $\mathrm{U}$ (union), mais pas uniquement. (...)

2. des programmes de génération automatique de formes et de mots, permettant de générer, pour chaque entrée verbale, ses formes conjuguées, ses dérivés (...) et, le cas échéant, le mot de base sur lequel le verbe est formé. (...) (Dubois et DuboisCharlier 1997b : p. XI)

Il est à noter que les opérations de tris croisés s'effectuent facilement à partir d'un tableur de type Excel ou OpenOffice ou, mieux, à partir des versions XML et JSON créées par Guy Lapalme ( $c f$. l'article de Lapalme et Le Pesant dans ce numéro). Les auteurs évoquent la génération des formes (cf. citation supra), mais l'opération de reconnaissance et d'annotation des mêmes formes est elle aussi possible à condition de disposer pour ce faire d'outils de TAL appropriés.

Les tables du lexique-grammaire du LADL étaient formatées en vue du traitement automatique. Dubois et Dubois-Charlier ont voulu qu'il en soit de même avec LVF. Maurice Gross avait confié à Max Silberztein la tâche de construire un logiciel de traitement de corpus, INTEX. Après la mort de Maurice Gross (2001), Silberztein crée et développe le logiciel $\mathrm{NooJ}^{8}$. Les deux composantes de base de NooJ sont des dictionnaires procurés par l'utilisateur (par exemple la version NooJ de LVF) et des grammaires locales en forme d'automates finis, construites elles aussi par l'utilisateur. NooJ est un outil approprié pour l'implémentation de LVF pour des tâches de reconnaissance de mots, de constructions et de phrases dans de grands corpus, ainsi que pour des tâches de fouille de textes et d'annotation sémantique. Ce logiciel permet d'intéressantes opérations de linguistique de corpus à partir des dictionnaires informatisés de Dubois et DuboisCharlier ( $c f$. l'article de Lapalme et Le Pesant dans ce numéro).

\section{Jean Dubois et Françoise Dubois-Charlier morphologues}

37 Dès le début de sa carrière de linguiste, Jean Dubois s'intéresse de près à la morphologie, puisqu'elle est le sujet de sa thèse secondaire sur les mots construits ${ }^{9}$ :

Dubois, J. (1962). «Etude sur la dérivation suffixale en français moderne et contemporain. Essai d'interprétation des mouvements observés dans le domaine de la morphologie des mots construits ». Thèse complémentaire pour le doctorat ès lettres, Paris, Larousse : Paris (118 pages) 
On relève, à la même époque, les publications suivantes :

Dubois, J., Guilbert, L., Mitterand, H. et Pignon J. (1960). « Le mouvement général du vocabulaire français de 1949 à 1960 d'après un dictionnaire d'usage ", in Le Français moderne, juillet 1960, pp. 86-107, 196-211. [Repris dans Dubois, Jean \& Dubois, Claude. (1971). Introduction à la lexicographie, Paris, Larousse, 208 pages]

Dubois, J. et Guilbert, L. (1961a). "La notion de degré dans le système morphologique du français moderne ", Journal de psychologie, pp. 57-64.

Dubois, J. et Guilbert, L. (1961b). «Formation du système préfixal intensif en français moderne et contemporain ", avec L. Guilbert, Le Français moderne, pp. 87-112.

Dubois, J. (1965a) et (1965b). « Grammaire transformationnelle et morphologie », Le Français moderne, 1965, avril, pp. 81-96, et juillet, pp. 178-187.

Dubois, J. (1966). «Essai d'analyse distributionnelle du verbe (les paradigmes de la conjugaison) », Le français moderne 34. pp. 185-209.

Selon la morphologue Florence Villoing, professeure à l'Université de Paris Nanterre (communication personnelle), «les travaux de Dubois sont systématiquement cités parce qu'ils sont certainement les premiers à avoir proposé un aperçu global de la morphologie du français et à avoir avancé des hypothèses, notamment sur les nominalisations, qui sont encore discutées aujourd'hui ». C'est le cas par exemple de Swiggers (2000), qui dans un bilan d'un siècle de morphologie française, fait deux longues citations de Dubois (1962). Voici une partie du jugement de Paul Swiggers sur la thèse complémentaire de Dubois sur la suffixation :

Ce travail, basé sur le mouvement du vocabulaire entre 1906 et 1961, est riche en données et en principes théoriques. On n'y trouve pas de segmentation proprement morpho(phono)logique ni une étude des alternances; l'intérêt du travail réside donc moins dans l'analyse morphologique et plutôt dans les aspects proprement lexicaux (richesse, diversification, fréquence et valeur d'emploi des éléments suffixaux). Selon Dubois, l'ensemble des affixes forme un système; chaque affixe se définit par les rapports qui l'unissent à la base et le différencient des autres affixes (...). (Swiggers 2000, § 62).

De fait, la thèse complémentaire de Jean Dubois (1962) est jusque aujourd'hui fréquemment citée comme représentative d'un renouveau des études en morphologie, à une époque où commencent à se diffuser en France le distributionnalisme et le générativisme américains.

41 Parmi les premiers articles que Dubois consacre à la morphologie, il y en trois (1960, 1961a et 1961b) qui sont en collaboration avec Louis Guilbert (1912-1977). Il est intéressant de constater que, dans une certaine mesure, Jean Dubois et Louis Guilbert non seulement partagent les mêmes convictions idéologiques et scientifiques, mais aussi connaissent des circonstances biographiques analogues : une pratique militante du syndicalisme dans l'enseignement secondaire, une entrée relativement tardive dans l'enseignement supérieur et un engagement fort auprès des Editions Larousse ${ }^{10}$. Selon Jacqueline Bastuji (1979), "Guilbert s'inscrivait dans la double tradition de la lexicologie historique comme discipline socio-linguistique, et de la lexicologie structurale ». Le terme de «lexicologie structurale » est sans doute un peu vague; il serait plus juste de dire que, comme Dubois, Guibert a d'abord été séduit par les nouvelles écoles américaines, transformationnalistes et générativistes. Ce qui est le plus intéressant dans la phrase de Bastuji, c'est la référence à la socio-linguistique: Dubois comme Guilbert entendent associer, dans la description des phénomènes d'affixation et de composition, les méthodes des grammaires transformationnelles avec la prise en 
compte des facteurs socio-économiques; cela, du reste, les distingue du mouvement structuraliste. Par exemple, dans Dubois et Guilbert (1961b : 101-103), les deux auteurs mettent en évidence le fait que le développement du système préfixal intensif (archi-, extra-, ultra-, sur-, super-, hyper-) est une réponse à des besoins lexicaux nouveaux en biologie, médecine, psychologie, mathématiques etc.

Dans son ouvrage sur La créativité lexicale (1975), Guilbert résume de la sorte un point de vue qu'il partage avec Dubois et dont il attribue la paternité à Darmesteter (1894) :

La transformation du schéma phrastique, générateur de la formation préfixée ou composée, est liée à l'activité langagière du locuteur contemporain. [...] Au principe d'analyse fondé sur l'origine historique des éléments composants, il convient de substituer le concept de transformation lexicale fondé sur l'équivalence entre un schéma phrastique d'énoncé et une construction lexicale instituant la possibilité de bifurcation à volonté vers le syntagme lexical ou vers la paraphrase (un instrument qui pèse les lettres > un pèse-lettres) (Guilbert 1975, cité par Hugues Galli (2013, note 35)

Vers la fin de leur vie, Jean Dubois et Françoise Dubois-Charlier ont eu à cœur de retourner aux questions de morphologie en produisant les ouvrages suivants, prochainement en ligne sur le site de MoDyCo :

Dubois, J. et Dubois-Charlier, F. (1999). La dérivation suffixale en français. Paris : Nathan.

Dubois, J. et Dubois-Charlier, F. (2000). Dictionnaire électronique des suffixes. Aix-enProvence : chez les auteurs.

Dubois, J. et Dubois-Charlier, F. (2001). Composition et préfixation en français, Aix-en-

Provence : chez les auteurs.

\section{Dubois et Dubois-Charlier lexicologues et lexicographes, avant les dictionnaires informatisés des dernières années}

J'évoquerai infra dans la Section 6.2 les derniers travaux lexicographiques de Dubois et Dubois-Charlier qui, du seul fait d'être formatés pour les applications au TAL, tranchent radicalement avec les dictionnaires plus traditionnels dont il va être maintenant question. Jean Dubois en a produit beaucoup, parfois en collaboration avec Françoise Dubois-Charlier, avec son frère Claude Dubois et avec d'autres linguistes.

Jean Dubois a dirigé, pour la partie langue française, une des équipes qui ont élaboré le Grand dictionnaire encyclopédique Larousse (1980-1985), appelé par la suite Grand Larousse universel. Je ne m'attarderai pas sur cet ouvrage monumental, et je me concentrerai sur ses dictionnaires (en collaboration pour certains avec Françoise Dubois-Charlier) fondés sur les principes de base des grammaires distributionnelles. On constate qu'ils ont un caractère expérimental. Ce sont :

le Dictionnaire du français contemporain. Dubois J., R. Lagane et alii 1967 ; 33000 mots

le LEXIS. Sous la direction de Dubois J. 1975 ; 76000 mots

le Dictionnaire du français langue étrangère, Niveau 1. Dubois J. et F. Dubois-Charlier. 1978.

le Dictionnaire du français langue étrangère, Niveau 2, Dubois J. et F. Dubois-Charlier. 1979. 


\subsection{Le Dictionnaire du français contemporain}

La parution du Dictionnaire du français contemporain (DFC) (Dubois, Lagane et alii 1967) fut un véritable événement éditorial, dans le grand public et auprès des universités. Pendant les années 1970 et 1980, il tint lieu de manuel pour l'étude du français dans les facultés de lettres. Voici la manière argumentée dont Jean Pruvost (2003) caractérise le DFC :

Le DFC : Une expérience distributionnaliste.

En quoi ce dictionnaire introduit-il une véritable révolution dans le genre ? La description en synchronie de 25000 mots, le dégroupement homonymique des unités lexicales (le cœur, l'organe, et le cœur, synonyme de sentiments, considérés comme des homonymes parce que de distribution différente), le regroupement morphologique (charger, chargement, décharger, surcharger regroupés dans le même article), la présence systématique d'exemples forgés, l'indication du degré de synonymie à l'aide de flèches ascendante ou descendante, la mention des niveaux de langue, ce sont là autant de principes qui, appliqués de manière rigoureuse et scientifique tout au long de l'ouvrage, lui donnent une notoriété inattendue. L'avancée méthodologique était considérable, et le fait qu'il s'agissait d'un petit dictionnaire destiné à des apprenants déterminait le franchissement d'une étape essentielle : de la dictionnairique de la réduction, on passait à la lexicographie heuristique ${ }^{11}$.

Dans une tonalité très proche de celle de Jean Pruvost, Claude Kannas (1996) porte ce jugement sur le DFC :

La grande innovation du Dictionnaire du français contemporain a été de n'être pas seulement un dictionnaire de décodage mais un dictionnaire qui aide à la production. (...). Le Dictionnaire du français contemporain se situe donc à la conjonction de la recherche linguistique, des souhaits de pédagogie nouvelle et des demandes de l'étranger pour les apprenants en français langue étrangère.

Claude Kannas loue également une autre innovation de Dubois : la prise en compte des unités polylexicales en tant que lexèmes à part entière :

L'identification, le classement et le traitement des "locutions" permettaient d'aborder d'un œil neuf ces unités lexicales.

50 Au début des années 1970 une équipe des éditions Larousse dirigée par Jean Dubois s'attelle au projet du Lexis. L'ouvrage paraît en 1975. Il s'agit d'un grand dictionnaire du français classique et littéraire ouvert aux langues de spécialité. On y retrouve les caractéristiques du DFC énumérées par Jean Pruvost (cf. citation supra), à ceci près qu'il comporte des exemples littéraires à côté des exemples forgés, ainsi que des notations étymologiques et des datations en fonction du sens.

\subsection{Le Dictionnaire du français langue étrangère de Dubois et Dubois-Charlier}

On vient de voir que Jean Pruvost et Claude Kannas insistent sur les intentions pédagogiques de Jean Dubois ${ }^{12}$ dans la conception du DFC. Elles président aussi, évidemment, à la publication des deux volumes du Dictionnaire du français langue étrangère, niveau 1 (1978) et niveau 2 (1979), avec Françoise Dubois-Charlier. Dans ces ouvrages se manifeste, outre la préoccupation pédagogique, celle de construire des catégories syntactico-sémantiques. Claude Kannas (1996 : 23) écrit à leur propos : 
Le Dictionnaire du français langue étrangère, niveau 1, paru en 1978, permettait d'acquérir une connaissance du vocabulaire et de la syntaxe de base du français au travers de phrases forgées et d'indications de constructions. Le Dictionnaire $d u$ français langue étrangère, niveau 2, paru en 1979, présentait pour la première fois à côté des innovations précédentes l'indication, pour les noms, de catégories sémantiques, ce qui permettait d'observer le fonctionnement syntaxique de ceuxci par classements d'objets. Ce dictionnaire est le premier à être accompagné d'une grammaire totalement nouvelle qui présente la syntaxe de ces différentes classes d'objets. Leeman, nous convenions que le Dictionnaire du français langue étrangère constitue une anticipation des travaux de Gaston Gross sur ce que ce dernier appelle des classes d'objets, c'est-à-dire des classes de mots cohérentes tant du point de vue syntaxique que sémantique (cf. Le Pesant et Mathieu-Colas 1998). Il ne semble pas que Gaston Gross ait eu connaissance du Dictionnaire du français langue étrangère de Dubois et Dubois-Charlier, non plus que de leurs dictionnaires informatisés, et le terme de classes d'objets employé par Claude Kannas n'est certainement pas une allusion aux travaux de Gaston Gross et de son équipe, le LLI. L'article de Guy Lapalme et Denis Le Pesant (dans ce numéro) reviendra de façon élargie sur le rapprochement entre les travaux de Dubois et DuboisCharlier et ceux de Gaston Gross.

\section{Dubois et Dubois-Charlier syntacticiens}

\subsection{Retour sur l'adhésion de Dubois et Dubois-Charlier au cadre théorique des grammaires distributionnelles et transformationnelles}

53 Jean Dubois et Françoise Dubois-Charlier (1970), s'inspirant du Chapitre 2 de Structures mathématiques du langage (Harris 1971 [1968]), synthétisent comme suit les thèses du distributionalisme, qui sont la source commune des grammaires de Harris, du LADL et de leurs propres travaux: les éléments de base de toute langue sont discrets, arbitraires, linéaires, et leurs combinaisons sont finies. A propos des combinaisons, les deux auteurs écrivent :

Toutes les combinaisons n'étant pas possibles, on peut donc définir les éléments grammaticaux comme les morphèmes par les restrictions qui sont imposées aux éléments qui les composent (les phonèmes), et on peut définir les phrases par les restrictions qui sont imposées aux éléments (morphèmes et ou suites de morphèmes) qui les composent. (...). Ce processus de classification permet de définir des classes de phonèmes, de morphèmes, de syntagmes et de phrases par les restrictions aux combinaisons possibles. L'objet de la description linguistique est d'abord de constituer ces classes et de faire une taxinomie. (Dubois et DuboisCharlier, $1970: 8$ )

Les propriétés qui distinguent les grammaires de Harris de celles de Chomsky sont nombreuses : le caractère algébrique des règles ${ }^{13}$, la mise en relation de la structure de la phrase avec son contenu informatif (par opposition à la théorie de la structure profonde) ; l'absence de règles de réécriture (d'où l'absence d'indicateurs syntagmatiques arborescents); le rejet de la structure SN-SV au profit de la structure Prédicat-argument(s) ; la lexicalisation de la tête propositionnelle (la tête de la Phrase n'est pas le symbole $P$, mais une entrée lexicale concrète); le rejet du métalangage artificiel au nom du principe que «la métalangue fait nécessairement partie de la 
langue »; et le fait, enfin, d'admettre dans la catégorie des prédicats une partie des prépositions, conjonctions et adverbes. Les propositions sont susceptibles d'endosser des formes alternatives, en faisant l'objet de paraphrases, ou transformations. Les deux principales opérations de ce type sont, dans les grammaires de Harris, les réductions (effacements et pronominalisations), les permutations d'arguments et les attachements, par exemple des affixes aux radicaux (cf. Harris 1976).

choix de Jean Dubois et de Françoise Dubois-Charlier d'inscrire leurs travaux dans le cadre théorique des grammaires de Harris n'a été complètement effectif, on l'a dit, que lorsque Jean Dubois se fut rapproché de Maurice Gross dans les années 1970, à une époque où émerge la réputation de ce dernier. Auparavant les travaux de Françoise Dubois-Charlier s'inscrivaient dans un cadre générativiste (cf. l'article de Jacques François dans ce numéro) ; quant à Jean Dubois, il s'était intéressé très tôt à Harris et l'avait cité dans sa Grammaire Structurale et dans bien d'autres écrits. Mais il était fatal que ce soit principalement à Maurice Gross que revienne le rôle de transplanter en France le distributionalisme américain dans sa version harrissienne: il était anglophone, non seulement linguiste mais aussi ingénieur et mathématicien, et après avoir eu une bourse à Harvard et y avoir rencontré au MIT Marcel-Paul Schützenberger ${ }^{14}$ qui aidait Chomsky à donner une forme mathématique correcte à ses grammaires, il s'était ensuite éloigné du générativisme pour se rendre à Philadelphie auprès de Harris. Titulaire d'une bourse du gouvernement français, il était là, en 1964, pour aider à son tour Harris à formaliser mathématiquement ses propres grammaires. Et c'est aussi là, comme le rapportent Chevalier et Encrevé, que tout se met en place :

Alors là, j'apprends la linguistique. J'en avais appris pas mal, finalement, mais ça ne s'était pas mis en place. Je n'avais pas fait de travail personnel sur le langage et, cette année-là, j'avais des cours particuliers de Harris (Déclaration de Maurice Gross à Chevalier et Encrevé 2006 : 259)

Par la suite, Gross invite Harris à donner un cours à l'Université de Vincennes; il le transcrit, le traduit en français et le publie sous le titre de Notes $d u$ cours de syntaxe (cf. Harris 1976). Maurice Gross, en fondant le LADL en 1972 à l'Université Paris 7 et en traçant le programme de construction d'un lexique-grammaire se situe dans la suite directe des travaux de Harris et il les complète sur une question essentielle. Les grammaires de Zellig Harris ont en effet le mérite d'être axiomatisées, mais leurs règles ont le défaut d'être privées de leur extension lexicale. Ce sera la tâche des chercheurs du LADL d'expliciter l'extension des règles en examinant la totalité des mots du français.

En adhérant dans une large mesure au programme du lexique-grammaire, Dubois et Dubois-Charlier impriment un cours nouveau à leurs recherches. Certes, Jean Dubois va animer au LADL des réunions sur les verbes, mais ce qui va sortir de ces réunions du point de vue des publications de Dubois et Dubois-Charlier, ce ne seront pas des tables supplémentaires du lexique-grammaire, mais LVF (Les Verbes français, 1997a et 1997b) et le DEM (Dictionnaire Electronique des Mots), deux dictionnaires informatisés dont le format est entièrement nouveau.

\subsection{Les deux dictionnaires informatisés de la dernière période}

58 Dans leurs dictionnaires informatisés, Les Verbes français (LVF) et le Dictionnaire Electronique des Mots (DEM), ce qu'apportent Dubois et Dubois-Charlier de novateur dans la tradition des grammaires distributionnelles et transformationnelles, c'est la mise en avant de la composante sémantique de la grammaire. Dans LVF, tant dans la version 
imprimée que dans la version informatisée, les classes les plus générales (dites classes génériques), au nombre de 14 , sont des classes syntactico-sémantiques :

Tableau 1 : Les 14 Classes génériques de LVF

\begin{tabular}{|l|l|l|l|}
\hline Code & Classe & Code & Classe \\
\hline C & Communication & $\mathbf{N}$ & Munir, démunir \\
\hline D & Don, privation & $\mathbf{P}$ & Psychologie \\
\hline E & Entrée, sortie & $\mathbf{R}$ & Mise dans tel ou tel état \\
\hline F & Frapper, toucher & $\mathbf{S}$ & Saisir, posséder \\
\hline H & Etat physique & $\mathbf{T}$ & Transformation \\
\hline L & Localisation & $\mathbf{U}$ & Union, réunion \\
\hline M & Mouvement & $\mathbf{X}$ & Verbes auxiliaires \\
\hline
\end{tabular}

Les auteurs présentent leur ouvrage sous un angle expérimental, en suggérant qu'il sert à la vérification d'une hypothèse sur la relation syntaxe-sens :

La classification des verbes français repose sur l'hypothèse qu'il y a adéquation entre les schèmes syntaxiques de la langue et l'interprétation sémantique qu'en font les locuteurs de cette langue : à la différence syntaxique entre venir à Paris et venir de Paris correspond une différence entre la destination et l'origine. (Préface de Les Verbes Français, page III)

L'hypothèse à vérifier n'est donc rien d'autre que celle de l'isomorphisme de la forme syntaxique et du sens, que Jean Dubois, dans la préface du tome 1 de sa Grammaire structurale du français, attribue à Harris, Hjelmslev et Bloomfield, et reprend à son compte (cf. supra Section 2.2). Cette théorie est du reste aussi présente dans les travaux du LADL ${ }^{15}$.

61 Les autres propriétés qui sont conservées dans les œuvres tardives de Dubois et DuboisCharlier par rapport aux lexiques-grammaires du LADL sont :

- la présence cruciale de la prise en compte de la syntaxe dans la description syntactico-sémantique, grâce à la rubrique Construction ;

- la méthode du dégroupement homonymique déjà mis en œuvre par Jean Dubois dans le Dictionnaire du français Contemporain (cf. supra Section 5.1), et poussé à l'extrême dans LVF (par exemple il y a 43 entrées pour prendre, 36 entrées pour tirer et 35 entrées pour tenir) ;

- le format tabulaire (pour la version informatisée de LVF) permettant l'implémentation informatique.

Les principales différences entre LVF et les lexiques-grammaires du LADL sont :

- la mise en avant, dans la rubrique Classe, des classes génériques qui, on vient de le voir, sont de nature sémantique ;

- la présence de la rubrique Opérateur, avec un jeu complexe de critères permettant d'affiner la classification syntactico-sémantique opérée par la rubrique Construction ${ }^{16}$; 
- la présence, grâce à la rubrique Sens, de (para-)synonymes qui explicitent encore davantage la dimension sémantique; par exemple la rubrique Sens du verbe conseiller 01 indique : « recommander, suggérer »;

- l'indication, dans la rubrique Domaine, des domaines pragmatiques, techniques, scientifiques etc., ainsi que le registre de langue ou les régionalismes (exemples: AER (aéronautique), ANA (anatomie humaine) ; les domaines mentionnés sont au nombre d'environ 120 ;

- les exemples forgés pour chaque entrée (exemple de cheminer 04: "L'entreprise chemine à sa perte. Ces idées cheminent lentement "); ils constituent à eux seuls un corpus textuel conçu de façon à ce qu'il ne comporte pas d'ambiguïtés.

On trouvera un examen plus approfondi de la structure de LVF dans François, Le Pesant et Leeman (2007). L'article de Guy Lapalme et de Denis Le Pesant (dans ce numéro) est consacré aux ultimes travaux de Dubois et Dubois-Charlier, d'une part Les Verbes Français (LVF), d'autre part le Dictionnaire Electronique des Mots (DEM), entreprise colossale malheureusement inachevée. Ces deux ressources sont téléchargeables sur le site de l'équipe de recherche MoDyCo (cf. http://www.modyco.fr/fr/Ressources/ ldlvf.html). Il en existe en outre des versions XML et JSON dues à Guy Lapalme (cf. http://rali.iro.umontreal.ca/rali/?q=fr/versions-informatisees-lvf-dem). Les deux linguistes, enfin, ont produit dans leur dernier article, Dubois \& Dubois-Charlier (2011), une description détaillée des principes et méthodes mis en œuvre dans le DEM.

\subsection{Une énigme : l'absence de la notion de verbes support dans Les Verbes Français (LVF)}

64 A côté des verbes prédicatifs, qui se caractérisent par le fait d'avoir une structure argumentale, c'est-à-dire le fait d'avoir un argument sujet et d'éventuels arguments compléments essentiels, il existe un ensemble de catégories de verbes non prédicatifs : les auxiliaires de conjugaison, les (semi-)auxiliaires aspectuels et modaux, les verbes supports des adjectifs et des noms, et les opérateurs causatifs (factitifs). Je me borne ici au cas des verbes supports. Les verbes supports, pour les définir en quelques mots, sont des verbes auxiliaires qui permettent aux prédicats nominaux et adjectivaux de se réaliser en phrases, comme le font de façon immédiate les prédicats verbaux. La notion est présente chez Harris ( $c f$. Ibrahim, A. 1996 : 4), et le terme apparaît dès les années 1970 chez quelques membres du LADL (M. Gross, A. Daladier) et fait l'objet d'un assez long développement dans Maurice Gross (1981: 32-45), qui distingue entre ceux qui sont les plus généraux (par exemple dans "avoir une excellente santé») et ceux qui sont plus spécifiques, appelés extensions de verbe support (par exemple dans « jouir d'une excellente santé »).

Compte-tenu des séances de travail fréquentes qu'animait Jean Dubois dans les murs au LADL dans les années 1981, de sa connaissance profonde des travaux de Harris et de Gross, et des références nombreuses qu'il y a fait ${ }^{17}$, il est impossible que Jean Dubois et Françoise Dubois-Charlier n'aient pas accepté la théorie des verbes-supports.

Or, dans Les verbes français (LVF) les verbes supports ne sont pas enregistrés. Plus exactement, ils y figurent mais au milieu des verbes prédicatifs, sans en être distingués ni reconnus comme verbes supports. Ils auraient pourtant eu toute leur place dans la classe X de LVF, qui est consacrée principalement aux autres sortes de verbes non prédicatifs, à savoir les auxiliaires de conjugaison, les auxiliaires aspectuels et modaux des verbes et des adjectifs, et les Opérateurs causatifs ${ }^{18}$. 
67 Le problème est le suivant. Comment se fait-il que les verbes supports ne soient pas enregistrés en tant que tels dans LVF?

Notre réponse découle d'abord d'un constat. Dubois et Dubois-Charlier ont élaboré LVF à une époque, les années 1980 et le début des années 1990, où l'existence de verbes supports était reconnue et où la notion était une pièce majeure dans la théorie des grammaires distributionnelles et transformationnelles, sans pour autant qu'on ait fait l'inventaire et l'analyse linguistique de ces verbes. En somme à cette époque, l'existence des verbes supports est reconnue, mais leurs propriétés spécifiques et leur effectif sont inconnus. Dans les années 1990, on évaluait couramment le nombre des verbes supports à quelques dizaines. On sait maintenant, en grande partie grâce aux travaux de Gaston Gross et son équipe, le LLI (CNRS et Université Paris 13) dans les années 1995-2005, qu'il faut plutôt l'évaluer à plusieurs centaines. Illustrons-le par l'exemple des quelque 740 noms de sentiments, sur lesquels je suis en train de travailler. J'ai relevé 72 verbes supports appropriés à cette catégorie lexicale ${ }^{19}$. En extrapolant (peut-être abusivement) cette proportion à l'ensemble des noms prédicatifs du français, on a une idée de l'extension considérable du vocabulaire des verbes supports, champ d'étude aujourd'hui encore largement inexploré.

Notre hypothèse sur l'absence des verbes supports dans LVF découle de ce constat. La voici : Dubois et Dubois-Charlier, ne pouvant pas prendre en charge à eux seuls le traitement de ce "continent inexploré », se seraient résolu à ignorer la notion au nom d'une sorte de « morale provisoire ».

\section{Conclusion}

70 Je tente maintenant de répondre brièvement à la question sous-jacente au titre de cet article. Quelle est la place de l'œuvre de Jean Dubois et Françoise Dubois-Charlier dans l'histoire de la linguistique? On manque de recul, quelques années seulement après leur mort, pour répondre de façon claire à cette question. Leurs productions sont-elles encore diffusées ? La réponse ne peut être que mitigée. Le dictionnaire Lexis semble être le seul de leurs ouvrages qui soit encore disponible en librairie; le dictionnaire papier Les Verbes français n'est plus diffusé par l'éditeur, mais sa version en format PDF librement accessible en ligne a fait l'objet de nombreux téléchargements. J'estime que le Dictionnaire du français contemporain (1967) mériterait d'être réédité et utilisé de nouveau, comme manuel de référence en quelque sorte, dans les départements universitaires de lettres et de linguistique, ainsi que le Dictionnaire du français langue étrangère (1978 et 1979). Quant aux dictionnaires informatisés accessibles en ligne, LVF et DEM, ils restent encore trop méconnus ( $c f$. dans ce numéro, l'article de Guy Lapalme et Denis Le Pesant).

71 Le reste de la production de Dubois et Dubois-Charlier, immense, appartient nécessairement à l'histoire. Mais cette œuvre s'inscrit-elle dans un courant bien déterminé dans l'histoire de la linguistique ? C'est le cas à mes yeux : les travaux de Dubois et Dubois-Charlier s'inscrivent clairement dans la lignée de ceux de Harris et de Maurice Gross. Leur apport principal dans cette tradition des grammaires distributionnelles et transformationnelles consiste à mon avis en trois choses: le raffinement dans la description syntaxique que permet un usage poussé de la méthode $\mathrm{du}$ dégroupement homonymique (qui rend compte de façon simple du phénomène de la polysémie); la mise en évidence du poids énorme de la polylexicalité dans la langue 
(mots composés, locutions, phrases figées) ; et la mise au premier plan de la sémantique dans l'activité de description syntactico-sémantique.

L'œuvre de Jean Dubois et de Françoise Dubois-Charlier aura-t-elle des continuateurs? Je le crois car, si remarquable que soit son ampleur, elle comporte, on l'a vu, un certain caractère d'inachèvement. Je ne veux pas dire par là qu'il faudrait compléter LVF en y ajoutant les verbes supports et les locutions verbales, ni qu'il faudrait corriger et terminer le DEM. Je veux dire seulement ma conviction que l'école des grammaires distributionnelles transformationnelles, à laquelle ont appartenu aussi Gaston Gross et ses collègues du LLI, reviendra un jour en syntaxe sur le devant de la scène, pour une continuation du travail entamé.

\section{BIBLIOGRAPHIE}

Bastuji, J. (1979). « Néologie et lexicologie (hommage à Louis Guilbert) », pp. 12-20, Paris : Larousse.

Chevalier, J.C. et Encrevé, P. (2006). Combats pour la linguistique, de Martinet à Kristeva. Lyon : ENS Editions.

Dubois, J. (1962). Le vocabulaire politique et social en France de 1869 à 1872 à travers les œuvres des écrivains, le revues et les journaux. Thèse de doctorat. Paris : Larousse.

Dubois, J. (1962). Etude sur la dérivation suffixale en français moderne et contemporain. Essai d'interprétation des mouvements observés dans le domaine de la morphologie des mots construits. Thèse complémentaire pour le doctorat ès lettres. Paris : Larousse, 118 pages.

Dubois, J. (1965-1969). Grammaire structurale du français. 3 volumes. Paris : Larousse.

Dubois, J. dir. (1972 a). Langue française, $\mathrm{n}^{\circ} 14,1972$. Linguistique, formation des enseignants et enseignement supérieur. Paris : Larousse.

Dubois, J. (1972 b). « Grammaire linguistique et grammaire pédagogique », Langue française $\mathrm{n}^{\circ} 14$. Paris : Larousse. 28-31

Dubois J. dir. (1975). LEXIS, le dictionnaire érudit de la langue française (76 000 mots). Paris : Larousse. Dubois, J., Dubois, Cl. (1971). Introduction à la lexicographie. Paris : Larousse, 208 pages Dubois, J. et Dubois-Charlier, F. (1970). Nouvelle grammaire du français Paris : Larousse. Dubois, J. et Dubois-Charlier, F. (1978). Dictionnaire du français langue étrangère Niveau 1. Paris : Larousse

Dubois, J. et Dubois-Charlier, F. (1979). Dictionnaire du français langue étrangère Niveau 2. Paris : Larousse.

Dubois, J. et Dubois-Charlier, F. (1997a). Les verbes français (LVF). Version informatisée (environ 26000 entrées). Version LVF +1 (Micosoft-Excel) : https://www.modyco.fr/fr/basedocumentaire/ressources/jean-dubois.html. Versions JSON et XML : http://rali.iro.umontreal.ca/ rali/?q=fr/versions-informatisees-lvf-dem

Dubois, J. et Dubois-Charlier, F. (1997b). Les Verbes français (428 pages). Paris : Larousse. 
Dubois, J., et Dubois-Charlier, F. (2008). Le nombre en français, avec un avant-propos d'Antoinette Balibar-Mrabti. Fernelmont/Paris : Editions Modulaires Européennes/L'Harmattan.

Dubois, J. et Dubois-Charlier, F. (2011). « La combinatoire lexico-sémantique dans le Dictionnaire Electronique des Mots. Les termes du domaine de la musique à titre d'illustration », in Leeman et Sabatier (éds), Langages n 179-180. Paris : Armand Colin. 31-56.

Dubois, J. et Dubois-Charlier, F. (2020). Dictionnaire Electronique des Mots (DEM). Version MicosoftExcel : https://www.modyco.fr/fr/base-documentaire/ressources/jean-dubois.html. Versions JSON et XML : http://rali.iro.umontreal.ca/rali/?q=fr/versions-informatisees-lvf-dem

Dubois, J., Guilbert, L., Mitterand, H. et Pignon J. (1960). « Le mouvement général du vocabulaire français de 1949 à 1960 d'après un dictionnaire d'usage ", in Le Français moderne, juillet 1960, pp. 86-107, 196-211. [Repris dans Dubois, Jean \& Dubois, Claude. (1971). Introduction à la lexicographie. Paris : Larousse, 208 pages

Dubois, J., Guilbert, L. (1961)a. « La notion de degré dans le système morphologique du français moderne », in Journal de psychologie, mars 1961, pp. 57-64.

Dubois, J., Guilbert, L. (1961)b. « Formation du système préfixal intensif en français moderne et contemporain », in Le Français moderne, avril 1961, pp. 87-112.

Dubois, J., Lagane R. et alii (1967). Dictionnaire du français contemporain. Paris : Larousse.

Dubois, J., Lagane, R. (1973). La nouvelle grammaire du français, Paris : Larousse

Dubois, J., Lagane, R. (1973) éd. Comment apprendre la grammaire (3 livrets), avec Frank Marchand, Danielle Leeman, Claudine Fabre, Annette Schutte. Paris : Larousse.

Dubois, J., Sumpf, J. éd. (1970) Langue Française n 5. Linguistique et pédagogie. Paris : Larousse.

Dubois-Charlier, F. (1970). Étude Neurolinguistique de l'Alexie Pure, Thèse. Faculté des Lettres et Sciences Humaines. Paris: Paris-Est.

Dubois-Charlier, F. (1976). « Les analyses neuropsychologiques de l'alexie : 1838-1969 », Langages $\mathrm{n}^{\circ} 44$, in H. Kremin \& F. Dubois-Charlier (éds.) Les troubles de la lecture : l'alexie, pp. 20-62

François, J., Le Pesant, D. et Leeman, D. (2007). «Présentation de la Classification des Verbes Français, de Jean Dubois et Françoise Dubois-Charlier », in Langue Française $n^{\circ} 153$. Paris : Larousse.

Galli, H. (2013). Echappée en morphologie dérivationnelle: approche épistémologique de la discipline avec application au préfixe français en. Thèse de doctorat. Université de Zürich.

Guilbert, L. (1975). La créativité lexicale. Paris : Larousse.

Harris, Z. S. (1952). Discourse Analysis, Language 28

Harris, Z. S. (1971) [1968]). Structures mathématiques du langage. Traduction par Catherine Fuchs de Harris, Z. S. [1968].

Harris, Z. S. (1976). Notes du cours de Syntaxe. Paris : Le Seuil.

Harris, Z.S. (1990). « La genèse de l'analyse des transformations et de la métalangue », in Les grammaires de Harris et leurs questions (Daladier, éd.), Langages n 99. Paris : Larousse.

Hécaen, H. (1972). Introduction à la Neuropsychologie. Paris: Larousse.

Ibrahim, A. H. (1996). Les supports, le terme, la notion, les approches, in Langages $n^{\circ} 121$. Paris :

Larousse. 3-7.

Irigaray, L. (1973). Le Langage des Déments. The Hague : Mouton. 
Jakobson, R., et Halle, M. (1956). Fundamentals of Language. La Haye (Netherlands): Masson et Cie.

Kannas Claude (1996). Jean Dubois et la lexicographie, in LINX n 34-35, Lexique, syntaxe...automatique. Hommage à Jean Dubois. Université Paris Nanterre. 21-25.

Le Pesant D., Mathieu-Colas M. (1998). « Introduction aux classes d'objets », in Langages $\mathrm{n}^{\circ} 131$. Paris : Larousse. 6-33.

Maingueneau, D. (1996). «Jean Dubois et les débuts de l'analyse du discours en France : quelques réflexions ", in LINX, n 34-35. Hommage à Jean Dubois. Nanterre : Université Paris 10. 27-33

Marchand, F. (1996). «Jean Dubois et la pédagogie », in LINX n 34-35. Nanterre : Université Paris 10. $45-54$

Marcie, P. (1996). « Jean Dubois et l'avènement de la neurolinguistique ». In LINX n 34-35. Nanterre : Université Paris 10. 35-44.

Marcie, P., Roudier, M., et Boiler, F. (1994). « Spontaneous language and impairment of communication in Alzheimer's disease ». Linguistische Berichte. Special Issue: Neurolinguistics 6. 111-127.

Pruvost, J. (2003). « Les dictionnaires français monolingues d'apprentissage: une histoire récente et renouvelée ». Quaderni del CIRSIL - 2 (2003) - www.lingue.unibo.it/cirsil.

Sabatier, P., Le Pesant, D. (2013). « Les dictionnaires électroniques de Jean Dubois et Françoise Dubois-Charlier et leur exploitation en TAL », in Ressources Lexicales, Chapitre 5, N. Gala et M. Zock ed. Amsterdam: John Benjamins.

Silberztein, M. (2015). La formalisation des langues. L'approche de NooJ. London : Iste Editions.

Swiggers, P. (2000). « Le champ de la morphologie française : Bilan des études et perspectives de recherche », in Modèles Linguistiques 42 (Un siècle de linguistique en France : morphologie et syntaxe). 14-32.

\section{NOTES}

1. Dubois indique à Chevalier et Encrevé (2006) qu'il n'y avait guère que les spécialistes de la grammaire comparée des langues européennes anciennes (Chantraine, Vendryes, Perrot et Lejeune) qui l'intéressaient, à cause de leur côté "formaliste ", en quelque sorte structuraliste avant la lettre. C'est ainsi qu'il loue Chantraine dans ce propos non dénué d'humour: "Jamais Chantraine n'a eu l'idée de commenter, même de loin, le rapport entre le texte homérique et le sens du texte homériques; cela ne l'intéressait absolument pas » (Chevalier et Encrevé, 2006:148).

2. Maître de Conférences à Tours puis Professeur des Universités à l'Université de Nanterre.

3. L'émergence de la philosophie analytique au Royaume Uni, aux USA et, de façon plus atténuée, en France, notamment dans l'analyse et la critique du langage ordinaire, est un autre exemple de linguistic turn. La source en est le deuxième Wittgenstein et le Cercle de Vienne, notamment Carnap qui, du reste, exerça une certaine influence sur Z. Harris (cf. Harris 1990: 12 et 19).

4. Par exemple, Dominique Maingueneau évoque les «problématiques lacano-althusseriennes d'un Michel Pêcheux ».

5. Cf. Harris Z. (1999: 9) dans Langages 99 et Maurice Gross (1981:7-52) dans Langages 63.

6. Cf. Jakobson (1961) [1941]. Langage enfantin et aphasie. Paris : Editions de Minuit ; R. Jakobson [Jakobson, 1941; Jakobson, 1956; Jakobson, 1964; Jakobson, 1968] ; cf. aussi Jakobson et Halle 1956 (Fundamentals of Language). 
7. Dubois (1972) écrit par exemple dans Langue Française $n^{\circ} 14$ : « Sur le plan de l'enseignement, le discours pédagogique se travestit parfois en un discours scientifique, élevant la grammaire scolaire des enfants de $6^{\mathrm{e}}$ à la hauteur d'une linguistique avancée. On érige la somme des résultats divers de théories différentes en une sorte de description éclectique dont on n'assure pas les bases théoriques, puisque celles-ci ne sont pas explicites ».

8. Sur NooJ, cf. Silberztein 2015 ainsi que, dans ce numéro, l'article de M. Silberztein et l'article de Lapalme et Le Pesant. Le logiciel est téléchargeable à partir de http://www.nooj-association.org/.

9. Jean Dubois utilise dès 1962 le terme de mots construits qu'imposeront 25 ans plus tard Danielle Corbin et ses disciples.

10. Il existe une longue notice sur Louis Guilbert dans le " Maitron » (Dictionnaire biographique du mouvement ouvrier et du mouvement social : https://maitron.fr/spip.php?article75506). Ce dernier fut un des dirigeants communistes du Syndicat National de l'Enseignement Secondaire (SNES) de 1944 à 1961. Il fut professeur de lycée, puis à partir de 1960, maître de conférences à Rouen, et à partir de 1968 professeur à l'université Paris 10 Nanterre. Sa thèse porte sur le vocabulaire de l'aviation. C'est un terminologue, un lexicologue et un morphologue, spécialisé notamment dans le domaine de la créativité lexicale. Il fut un des premiers linguistes à s'intéresser à la néologie. En tant que lexicographe, il n'est rien moins que co-auteur, avec René Lagane et Georges Niobey, du Grand Larousse de la Langue Française (GLLF, 1971-1977).

11. Les soulignements sont de mon fait.

12. Sur ce point on pourra consulter l'article de F. Marchand (1996)

13. Par opposition au caractère logique des règles de réécriture, selon Maurice Gross dans la préface de Notes du cours de syntaxe (Harris 1975).

14. Le médecin, généticien, statisticien, théoricien de l'information et mathématicien français M.-P. Schützenberger, reconnu comme un des créateurs de l'informatique théorique, fut un des plus proches amis de Maurice Gross.

15. Sur ce point, on se reportera à M. Gross (1981:20-21), pour qui il existe ce qu'il appelle un morphisme entre la forme syntaxique et la forme sémantique, c'est-à-dire « une redondance importante entre les deux ensembles, au point qu'on peut penser que l'un d'entre eux pourrait être inutile ». Dans ce passage, Gross s'autorise de Harris, en donnant trois références, dont Harris 1968. Dans LVF, les codages de propriétés sont pour l'essentiel de nature morphosyntaxique et la formalisation syntaxique relève dans les grandes lignes des théories de la grammaire distributionnelle et transformationnelle et du lexique-grammaire. D'autre part il n'y aucune formalisation sémantique dans LVF. Les notations explicitement sémantiques, à savoir les classes génériques et les synonymes de la rubrique Sens, ne servent à nos yeux qu'à rendre plus sensible l'existence de l'isomorphisme de la syntaxe et du sens.

16. Soit par exemple (cf. Les Verbes français (1997a), page 19) dans la Classe C (= les verbes de communication), la Sous-classe syntactico-sémantique $\mathrm{C} 2 \mathrm{~b}$ (= transitifs avec complétive en que + subjonctif ou une infinitive, avec instrumental (ex. par une circulaire on interdit aux élèves d'afficher). La rubrique Opérateur permet d'affiner la classification en distinguant conseiller 01 ( $\mathrm{OP}=$ dic conseil A qq D) de déconseiller 01 ( $\mathrm{OP}=$ dic conseil A qq D nég), ou en distinguant ordonner 04 (OP= dic ordre A qq D) de permettre 01 (OP= dic possible A qq D).

17. Il convient à ce propos de citer in extenso la dédicace qui ouvre Les Verbes français (1997): Nous tenons à remercier les membres du LADL (CNRS) de Maurice Gross et, en particulier, Alain Guillet, à qui cet ouvrage doit beaucoup, ainsi que nos collaborateurs des dictionnaires Larousse qui ont participé avec nous la rédaction du Dictionnaire du français contemporain, $d u$ Lexis, des Dictionnaires du français langue étrangère, de la partie langue du Grand Dictionnaire Encyclopédique Larousse.

18. Figurent tout au moins dans la Classe X de LVF la copule être, verbe support par excellence, et quelques extensions de cette dernière, ainsi que l'Opérateur factitif rendre. Figurent aussi des verbes supports de noms d'événements comme y avoir, exister et leurs variantes aspectuelles commencer et finir (ex. le spectacle est fini). Mais ces verbes supports ne sont pas reconnus en tant 
que tels dans la Classe X, et surtout, plusieurs centaines d'autres verbes supports sont éparpillés dans toutes les autres classes au milieu des verbes prédicatifs.

19. Les verbes supports précédant le nom prédicatif et passivables sont : éprouver, ressentir ; caresser, nourrir, concevoir (désir), couver (rancune), entretenir (chagrin) ; goûter (plaisir), sentir (apaisement); subir (humiliation), vivre, revivre (calvaire).

Les verbes supports précédant le nom prédicatif et non passivables sont: avoir (tristesse), connaître (désillusion); être plein de, être au comble de, déborder de (confiance); brûler, griller de (envie), brûler de (amour), bouillir de (colère) ; être bourrelé de (remords) ; être gonflé, bouffi de (orgueil) ; être pétri de (doute) ; jouir de (moral d'acier) ; se prendre, être pris de (affection); souffrir de (dépression); être en proie à (remords); vouer, porter à qq (admiration) ; être dans (bonheur, malheur), être dans, se mettre dans (colère) ; plonger dans, être plongé dans, sombrer dans (désespoir); nager dans (euphorie); $\boldsymbol{y}$ avoir entre $\mathrm{x}$ et $\mathrm{y}$ (tension).

Les verbes supports précédés du nom prédicatif, et passivables (à une exception près) sont: accabler, assaillir, harceler, poursuivre ; atteindre ; brûler, consumer, enflammer ; crucifier, tarauder, tenailler, torturer ; démanger, titiller ; dévorer, miner, ronger ; étreindre, oppresser ; envahir; frapper; gagner; glacer, tétaniser, paralyser; habiter, hanter ; prendre ; presser ; saisir ; submerger ; transporter; s'emparer de. Exemple: le regret de ne pas avoir épousé Marie taraude Pierre (= «Pierre a un (fort) regret de ne pas avoir épousé Marie » = " Pierre est taraudé par un (fort) regret de ne pas avoir épousé Marie »).

\section{RÉSUMÉS}

Cet article a pour ambition de situer l'œuvre de Jean Dubois (1920-2015) et Françoise DuboisCharlier (1942-2016) dans la lignée de ce qu'on pourrait appeler l'école française de grammaire distributionnelle et transformationnelle, qui découle des travaux de Z. Harris et dont les deux autres membres importants sont Maurice Gross et Gaston Gross. L'accent est également mis sur l'influence de leurs travaux sur la vie intellectuelle française des années 1960-1990, de par la variété et la puissance de leurs talents. En effet, leur domaine de recherche ne se limite pas à la syntaxe, il s'étend aussi à l'analyse du discours, à la morphologie, à la neurolinguistique, à la pédagogie du français, et surtout à la lexicologie et la lexicographie, y compris la lexicographie informatisée.

This paper positions the work of Jean Dubois (1920-2015) and Françoise Dubois-Charlier (1942-2016) in the tradition of the "French school of distributional and transformational grammar" along the lines of Z. Harris. Other prominent members of this trend were Maurice Gross and Gaston Gross. We emphasize the variety and power of their talent and show their influence on the French intellectual life from the sixties through the nineties. Their research was not limited to syntax, it spanned discourse analysis, morphology, neurolinguistics, French language teaching, but more importantly, it dealt with lexicography, whether computer-based or not. 
INDEX

Keywords : French linguistics, English linguistics, syntax, distributional and transformational grammars, Jean Dubois, Françoise Dubois-Charlier, discourse analysis, morphology, neurolinguistics, French teaching, English teaching, lexicology, computer-based dictionaries, Zellig Harris, Maurice Gross.

Mots-clés : Linguistique française, linguistique anglaise, syntaxe, grammaire distributionnelle et transformationnelle, Jean Dubois, Françoise Dubois-Charlier, analyse du discours, morphologie, neurolinguistique, enseignement du français, lexicologie, dictionnaires informatisés, Zellig Harris, Maurice Gross

\section{AUTEUR}

\section{DENIS LE PESANT}

Université de Paris Nanterre et CNRS MoDyCo 